\title{
Urban Trip Requests Prediction: An Operator's Perspective
}

\author{
Yong Tian ${ }^{+}$, Ningyuan Huang, Weidong Liu and Jiaxing Song \\ Department of Computer Science and Technology, Tsinghua University, Beijing, China
}

\begin{abstract}
With the help of e-hailing apps, such as Uber, finding passengers is no longer a problem for drivers. Transportation network companies' operators are concerned about how to allocate their cars in the urban area to maximize the company's profit. To answer that question, they have to know how trip requests are distributed over time and regions. In this paper, we present Gaussian Mixture Experts (GME), to partition the city into multiple regions and estimate of the trip request density with fixed Gaussian components but time-variant weights. We utilize the periodicity to predict the trip requests by context filtering. Our method is tested on real industrial urban trip request data. The result shows that our method achieves a good approximation to the real condition.
\end{abstract}

Keywords: trip request prediction, spatial data mining, Gaussian mixture experts, EM algorithm.

\section{Introduction}

In recent years, transportation network companies (TNC), such as Uber, have changed the taxi industry and the way people transport dramatically. For taxi drivers, it is much easier to find passengers by accepting trip requests using an e-hailing app than searching the city. Besides that, passengers do not need to stand by the roads, hailing taxis passing by. Instead, they push a button on their smartphones and get a ride in minutes.

Knowing the amount and distribution of trip requests at any specific time is very important for TNCs. First, it helps them to decide how to allocate car and driver resources to maximize the company's profit. On one hand, allocating more cars to regions with more trip requests will facilitate more deals and increase the income. On the other hand, it can also reduce the company's operating cost by minimizing empty driving. Second, many TNCs use surge pricing policy, they have to know when and where demand will surpass supply. Third, allocation according to request distribution reduces passengers' waiting time and leads to better user experience.

Some previous works [1]-[3] focus on how to find hotspots of high density of trip demands. They use clustering methods, such as DBSCAN [4], hierarchical clustering [5] and k-means [6], to cluster taxi trajectory data and find target places. These works mainly help drivers and passengers meet more easily, which is not a problem anymore due to e-hailing apps. Some other works [7]-[9] build statistical models to predict the amount of passengers at specific locations, such as taxi stands and roads, which are chosen in advance. All these works provide some information about the trip requests, but they are more useful to drivers or passengers rather than TNCs' operators. From an operator's perspective, the trip request number of a larger fixed region at a given time is of more interest, which is more convenient when deciding how to allocate cars over the urban areas to maximize the company's profit and minimize passengers' average waiting time.

\section{Approach}

\subsection{Problem Reduction}

\footnotetext{
+ Corresponding author. Tel.: + 8615201967560; fax: +861062781446.

E-mail address: gnoynait@gmail.com.
} 
Our target is to find a function $\eta(x, t)$ that $\int_{t \in \tau, x \in R} \eta(x, t) d x d t$ is the expectation of trip request number in region $R$ during time period $\tau$. Here, $x \in \mathbb{R}^{2}$ is geographical coordinates and $t \in \mathbb{R}$ is time. There are many factors that can influence $\eta$, such as region function (resident, commercial, etc.), population density, weather and e-hailing service price. These factors work in different ways. Some factors influence the distribution of the requests. For example, resident regions have more requests in the morning than in the evening while commercial districts may have the opposite condition. Other factors influence the total request number. When TNCs lower the service price, or the weather is bad, total request number may surge up, but the distribution of requests across the city may remain unchanged. Therefore, we assume $\eta(x, t)=$ $n(t) d(x, t)$, where $n(t)$ is a function which satisfies $\int_{t \in \tau} n(t) d t$ is the expectation of total request number in the whole area and $p(x, t)$ is the distribution density function of trip requests at time $t$.

It is hard to model $d(x, t)$ directly, therefore we resort to approximation. An appealing method is to partition the city into fixed regions and model the weight of each region over time, which is convenient for operators to allocate resources. We use time-variant mixture distribution $p(x, t)=\sum_{k} w_{k}(t) \mathcal{N}\left(x \mid \mu_{k}, \lambda_{k}^{-1}\right)$ to approximate $d(x, t)$. Here, $w_{k}(t), k=1,2, \cdots, K$, are $K$ functions of time $t$, satisfying $\sum_{k} w_{k}(t)=1$. Because $n(t)$ and $w_{k}(t)$ will not change dramatically in a short period $\Delta t$, we split the requests every $\Delta t$ time to form multiple groups. In each group $g$, we estimate the weight $w_{g}^{(k)}$ of Gaussian component $k$ and the request number $n_{g}$. After that, we use time series $n_{g}, w_{g}^{(k)}, g=1,2, \ldots, t, \ldots$, to approximate $n(t)$ and $w_{k}(t)$. In next section, we propose Gaussian Mixture Experts to estimate $w_{g}^{(k)}$ and learn $\boldsymbol{\mu}$ and $\boldsymbol{\lambda}$ from historical request data.

\subsection{Gaussian Mixture Experts}

Gaussian Mixture Experts (GME) is an extension of Gaussian Mixture Model (GMM) [10]. It estimates the densities of multiple groups of data with the same Gaussian components but different weights. Suppose there are $G$ groups of data, and we want to use $K$ Gaussian components to do density estimation. Each Gaussian component $k$ is parametric with mean $\mu_{k}$ and precision $\lambda_{k}$, which is drawn from a Gamma distribution with parameters $\alpha, \beta$. For data $x_{g n}$ in group $g$, draw a multivariate variable $z_{g n}$ from group $g$ 's weight $w_{g}$, and $x_{g n}$ is drawn from Gaussian distribution $\mathcal{N}\left(\mu_{z_{g n}}, \lambda_{z_{g n}}^{-1}\right)$. For convenience, we use vector representation of $z_{g n}$, in which $z_{g n}^{(i)}$ is 1 if $z_{g n}$ equals to $i$, otherwise $z_{g n}^{(q)}$ is 0 . The joint distribution for GME is

$$
p(\boldsymbol{X}, \boldsymbol{Z})=\prod_{g} \prod_{n} \prod_{k} \mathcal{N}\left(x_{g n} \mid \mu_{k}, \lambda_{k}^{-1}\right)^{z_{g n}^{(k)}} \operatorname{Mult}\left(z_{g n} \mid w_{g}\right) \operatorname{Gam}\left(\lambda_{g} \mid \alpha, \beta\right),
$$

where $\mathcal{N}\left(x \mid \mu, \lambda^{-1}\right)=\frac{1}{\sqrt{2 \pi \lambda}} \exp \left\{-\frac{\lambda}{2}(x-\mu)^{T}(x-\mu)\right\}, \operatorname{Mult}(z \mid w)=\prod_{k}\left(w^{(k)}\right)^{z^{(k)}}$ and $\operatorname{Gam}\left(\lambda_{g} \mid \alpha, \beta\right)=\frac{1}{\Gamma(\alpha)} \beta^{\alpha} \lambda^{\alpha-1} e^{-\beta \lambda}$. The probabilistic graph is shown in Fig. 1.

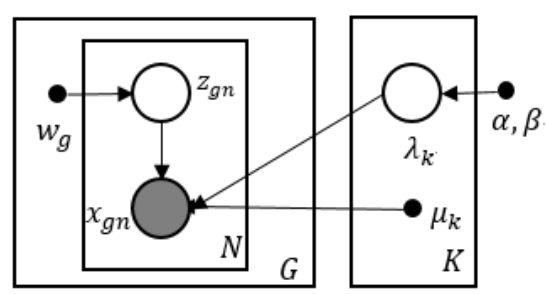

Fig. 1 Probabilistic Graph for GME.

To infer the best parameters for GME, we develop an EM algorithm [11] to do maximum a posterior (MAP) [12] inference, setting model parameters by $\left(\boldsymbol{w}^{*}, \boldsymbol{\mu}^{*}, \boldsymbol{\lambda}^{*}\right)=\operatorname{argmax}_{\mathbf{w}, \boldsymbol{\mu}, \boldsymbol{\lambda}} p(X \mid \boldsymbol{w}, \boldsymbol{\lambda}, \boldsymbol{\mu}) p(\boldsymbol{\lambda} \mid \alpha, \beta)$. Where $p(X \mid \boldsymbol{w}, \lambda, \boldsymbol{\mu})=\prod_{\mathrm{g}} \prod_{n} \sum_{k} w_{g}^{(k)} \mathcal{N}\left(x_{g n} \mid \mu_{k}, \lambda_{k}^{-1}\right)$ and $p(\lambda \mid \alpha, \beta)=\prod_{g} \operatorname{Gam}\left(\lambda_{k} \mid \alpha, \beta\right)$. By setting the derivative of parameters to zeros, we get the updating formulas:

$$
\begin{gathered}
z_{g n}^{(k)} \propto \mathrm{w}_{g}^{(\mathrm{k})} \mathcal{N}\left(x_{g n} \mid \mu_{k}, \lambda_{k}^{-1}\right) \\
w_{g}^{(k)}=\frac{\sum_{n} z_{g n}^{(k)}}{\sum_{n} \sum_{k} z_{g n}^{(k)}}, \quad \mu_{k}=\frac{\sum_{g} \sum_{n} z_{g n}^{(k)} x_{g n}}{\sum_{g} \sum_{n} z_{g n}^{(k)}}, \quad \lambda_{k}^{-1}=\frac{\beta+\sum_{g} \sum_{n} z_{g n}^{(k)}\left(x_{g n}-\mu_{k}\right)^{T}\left(x_{g n}-\mu_{k}\right)}{\sum_{g} \sum_{n} z_{g n}^{(k)}+\alpha-1}
\end{gathered}
$$


Below is the EM algorithm for GME:

1. Initialize parameters $\boldsymbol{w}, \boldsymbol{\mu}, \boldsymbol{\lambda}$ randomly;

2. Loop until convergence:

a) E step: estimate posterior distribution according to equation (2);

b) M step: update parameters according to equations (3);

\subsection{Trip Request Prediction}

Apply GME to historical trip request data, we achieves three goals in one step. First, we can partition the urban area into different regions by assigning coordinates to the most likely component. Second, we get region $k$ 's request number in period $g$, which is $n_{g}^{(k)}=n_{g} w_{g}^{(k)}$. Third, the density function in a period $g$ can be approximated by $p(x, g)=\sum_{k} w_{g}^{(k)} \mathcal{N}\left(x \mid \mu_{k}, \lambda_{k}^{-1}\right)$.

With historical estimation in hand, there are two ways to predict trip request's amount and distribution. The first one is using statistical regression models, such as [13][14] to learn a prediction function. The other approach is context filtering, which use historical data with similar context as prediction. The context includes time of day, day of week, weather and so on. In this paper, we choose the second strategy because of its simplicity and the strong weekly periodicity of trip requests data.

\section{Experiments}

\subsection{Dataset Description}

We get the trip request dataset from CAR Inc. ${ }^{1}$. It contains all trip requests of the company's urban transportation service from Sep. 1 to Sep. 30, 2015. Each record contains details of the requests, including the request's time and coordinate. We only keep records in a block ranging from $39.8 \mathrm{~N}$ to $40.0 \mathrm{~N}$ and from 116.3E to $116.5 \mathrm{E}$, which covers the centre of Beijing city. After filtering, there are about 800, 000 records left.

\subsection{Partition and Density Estimation Experiments}

Fig. 2 demonstrates the partition and density estimation results. We use 60 Gaussian components and set time period of each group to 30 minutes. In the left graph, each red circle corresponds to a Gaussian component with circle's center and radius as mean $\mu$ and variance $\lambda^{-1}$. The city is partitioned to multiple regions by most likely component. The blue lines are region boundaries. There are some small regions inside another region. Small region size indicate that region has a high density of trip requests. It turns out that, these small regions are train stations. For instance, the small circle in the middle of the left side is Beijing West Railway Station, one of the largest railway station in Asia.
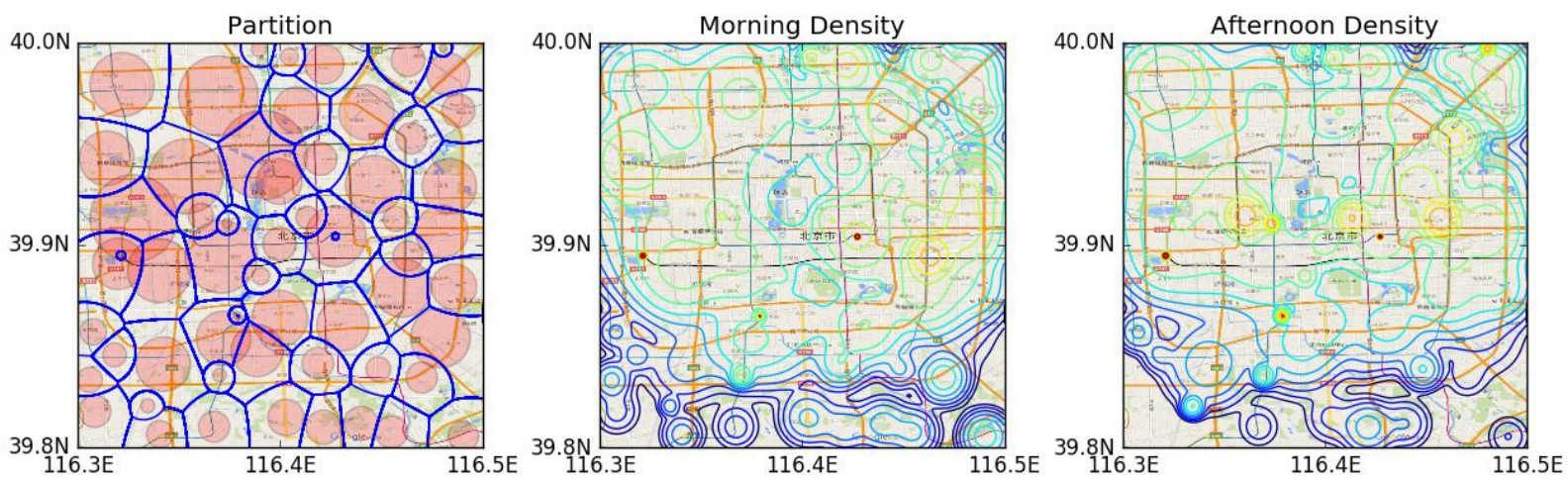

Fig. 2: Partition and density estimation results.

The other two graphs in Fig. 2 show the density of trip requests at 8:00 a.m. to 8:30 a.m. and 5:30 p.m. to 6:00 p.m., respectively. Redder contour line corresponds to higher density. The densest regions are railway stations with no doubt. The upper and middle part of the map is the center of Beijing, thus have a relatively high density. From these two, we can find that the lower part takes higher portion of requests in the morning

\footnotetext{
${ }^{1}$ China Auto Rental Inc. http://zhuanche.zuche.com/
} 
than in the afternoon, and some regions in the middle part have the opposite conditions. These are characteristics of residential and work area, respectively.

Fig. 3 shows the periodicity of trip requests in both amount and density. The left graph shows how request amount change every 30 minutes in a week. We can see that workdays' curve have a significant difference from weekends'. They have a valley at about 10 a.m. The middle and right graph show the weight and requests number in a week of three different regions. Region $\mathrm{B}$ and $\mathrm{C}$ correspond to Beijing Financial Street, a typical work area, and Jinsong, a typical residential area. The former have more request in the afternoon while the latter in the morning. Region C, called Sanlitun, is more interesting. It takes account of about $20 \%$ of requests of the whole city after midnight because of so many night clubs there. Although the proportion is high, we can see from the right graph that its request number is still small because of the small overall request number after midnight.
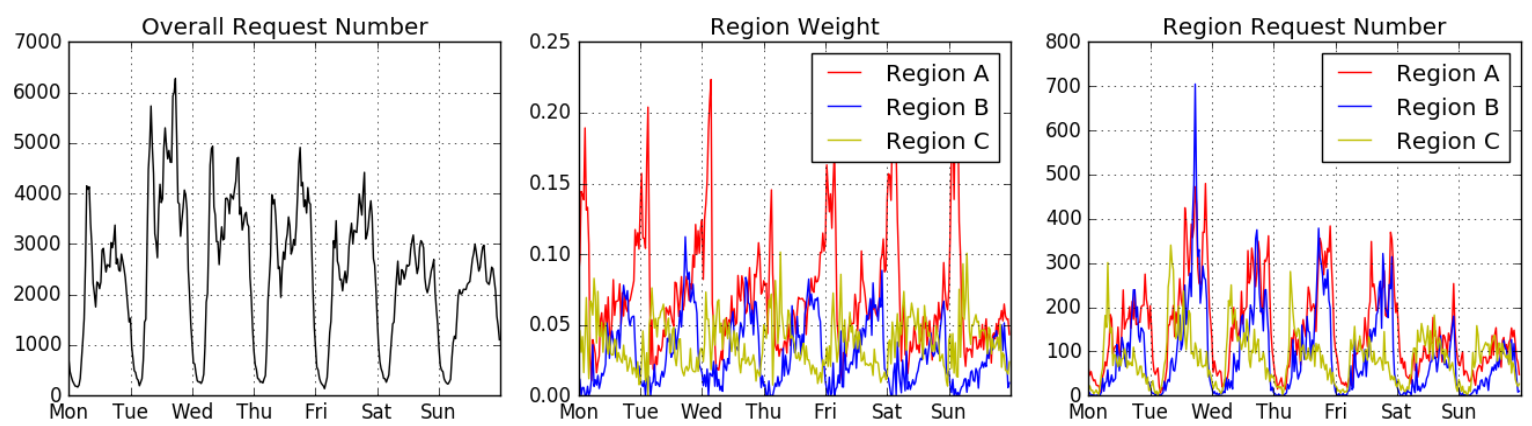

Fig. 3: Weekly periodicity of trip request.

\subsection{Prediction Performance}

Request number prediction is easy in context filtering. Thus we focus on the evaluation of density prediction performance. To achieve that goal, we leave one week's data out as test data, and fit GME on the other three weeks' data with $\Delta t$ set to one hour. After that, we evaluate the likelihood of test data every hour. High likelihood indicate good prediction performance. We repeat the process four times, each time with a different week as test data. As a comparison, we also fit a Gaussian mixture model (GMM) in the same setting. The results are shown in Fig. 4. The average of four experiment results are plotted against the time. In every hour, the data size is different, therefore the likelihood varies in a large range and has a strong periodicity. We can conclude from the graph that GME always have higher likelihood than GMM, which indicates GME has a better prediction performance than GMM.

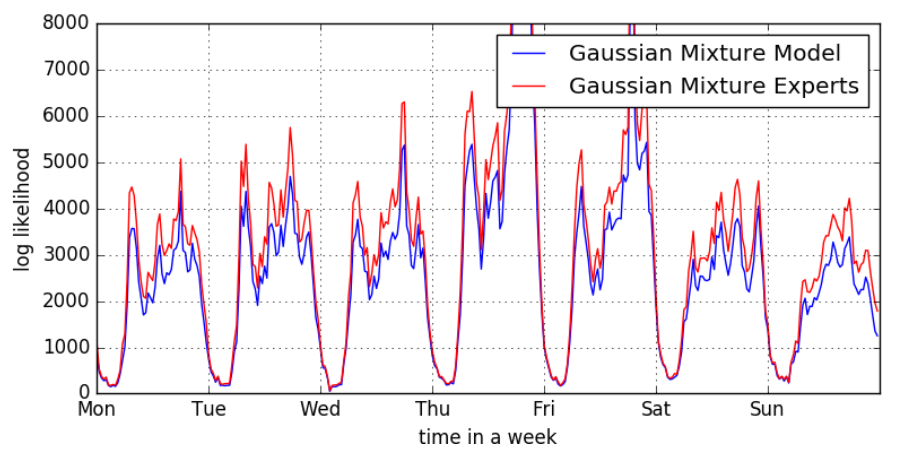

Fig. 4: Prediction Likelihood.

\section{Conclusion}

In this paper, we propose a method to predict urban trip requests in a specific region at a specific time. We reduce the problem into request amount prediction and request density prediction and use Gaussian mixture experts model to approximate the request density with fixed Gaussian components and time-variate weights. Experiments shows that, our method can partition the city into multiple reasonable regions and 
estimate their request proportions at different time. Our method can provide TNCs' operators important information to determine how to allocate their car and driver resources properly.

\section{References}

[1] Chang, H. W., Tai, Y. C., \& Hsu, J. Y. J. (2009). Context-aware taxi demand hotspots prediction. International Journal of Business Intelligence and Data Mining, 5(1), 3-18.

[2] Yue, Y., Zhuang, Y., Li, Q., \& Mao, Q. (2009, August). Mining time-dependent attractive areas and movement patterns from taxi trajectory data. In Geoinformatics, 2009 17th International Conference on (pp. 1-6). IEEE.

[3] Yuan, N. J., Zheng, Y., Zhang, L., \& Xie, X. (2013). T-finder: A recommender system for finding passengers and vacant taxis. Knowledge and Data Engineering, IEEE Transactions on, 25(10), 2390-2403.

[4] Ester M, Kriegel H P, Sander J, et al. A density-based algorithm for discovering clusters in large spatial databases with noise[C]//Kdd. 1996, 96(34): 226-231.

[5] Murtagh F. A survey of recent advances in hierarchical clustering algorithms[J]. The Computer Journal, 1983, 26(4): 354-359.

[6] Pelleg D, Moore A W. X-means: Extending K-means with Efficient Estimation of the Number of Clusters[C]//ICML. 2000, 1.

[7] Zheng, X., Liang, X., \& Xu, K. (2012, August). Where to Wait for a Taxi?. In Proceedings of the ACM SIGKDD International Workshop on Urban Computing (pp. 149-156). ACM.

[8] Moreira-Matias, L., Gama, J., Ferreira, M., Mendes-Moreira, J., \& Damas, L. (2012, October). Online Predictive Model for Taxi Services. In IDA (pp. 230-240).

[9] Moreira-Matias, L., Gama, J., Ferreira, M., Mendes-Moreira, J., \& Damas, L. (2013). On predicting the taxipassenger demand: A real-time approach. In Progress in Artificial Intelligence (pp. 54-65). Springer Berlin Heidelberg.

[10] Bishop C. Pattern Recognition and Machine Learning (Information Science and Statistics), 1st edn. 2006. corr. 2nd printing edn[J]. 2007, pp. 430, 439.

[11] Bilmes, J. A. (1998). A gentle tutorial of the EM algorithm and its application to parameter estimation for Gaussian mixture and hidden Markov models. International Computer Science Institute, 4(510), 126.

[12] Dellaert, F. (2002). The expectation maximization algorithm.

[13] Box, G. E., \& Pierce, D. A. (1970). Distribution of residual autocorrelations in autoregressive-integrated moving average time series models. Journal of the American statistical Association, 65(332), 1509-1526.

[14] Lee, S., \& Fambro, D. (1999). Application of subset autoregressive integrated moving average model for shortterm freeway traffic volume forecasting. Transportation Research Record: Journal of the Transportation Research Board, (1678), 179-188. 